\title{
From inside out to outside in
}

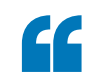

\section{compound}

uptake in

\section{M. tuberculosis}

by ATP-binding cassette $(A B C)$

exporters

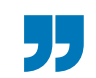

Multidrug-resistant Mycobacterium tuberculosis poses a great challenge for treatment and control strategies of tuberculosis worldwide, and improved diagnostics as well as new drugs and drug targets are urgently needed. Two papers now report compound uptake in M. tuberculosis by ATP-binding cassette (ABC) exporters, which are usually involved in the export of substrates out of the cell and could be new drug targets.

The papers provide structural insights into the mechanisms by which cobalamin (vitamin B12) and mycobacterial iron-binding siderophores are imported from the host environment, and blocking the import of these nutrients is a potential avenue for drug discovery.

Although M. tuberculosis can endogenously synthesize cobalamin, the pathogen also scavenges cobalamin from the host environment, and this process has been implicated in pathogenicity. No cobalamin transporter has been identified in M. tuberculosis, but reports have suggested that the

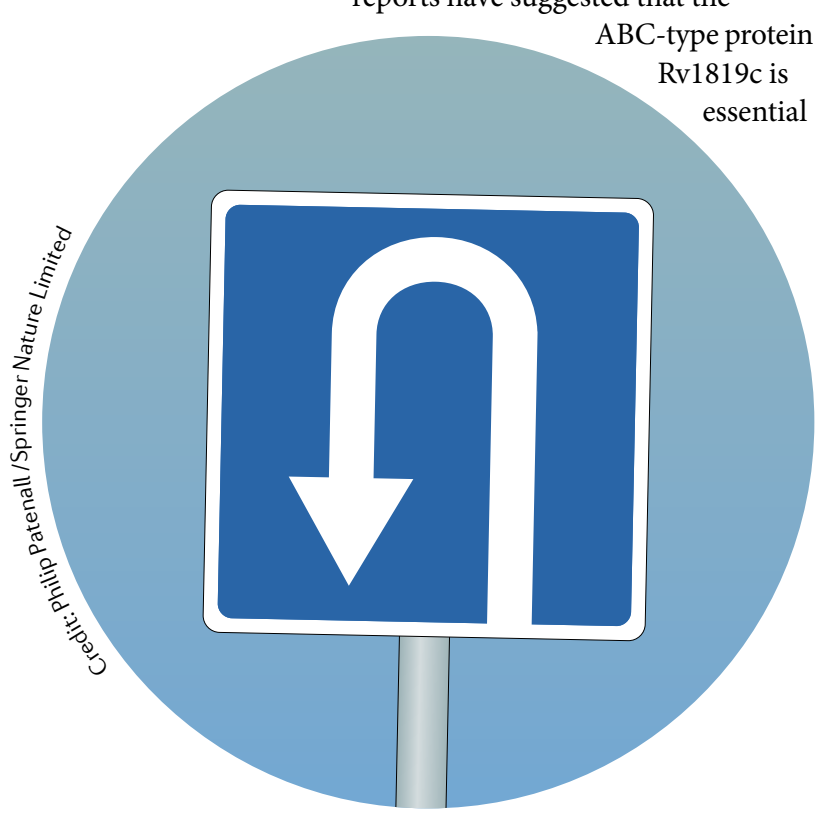

for cobalamin uptake, a protein that was previously shown to contain a bacterial ABC-exporter fold; a feature that seems to contradict an import function. In the first paper, Gati, Guskov, Slotboom and colleagues set out to elucidate the structural details involved in Rv1819c-mediated cobalamin import. Structural analysis revealed that the transporter is homodimeric, with each protomer consisting of a transmembrane domain fused to a nucleotide-binding domain (NBD), and the authors confirmed the presence of the previously identified bacterial ABC-exporter fold. Moreover, they also report that the protein contains an occluded cavity with a volume of more than $7,700 \AA^{3}$ that comprises two chambers, spans the entire thickness of the membrane and extends into the intracellular part of the protein. The inner surface of this cavity is lined with polar and negatively charged residues, which are favourable for interactions with hydrophilic molecules. The authors note that there is a lack of high-affinity substrate-binding sites and propose that the translocation chamber enables the transport of multiple structurally unrelated compounds. Indeed, they show that not only cobalamin but also the antimicrobial peptide bleomycin is transported. The authors suggest a model, whereby in the ATP-bound state the intracellular NBDs assume the closed dimer conformation and the extracellular gates can open, which enables substrate to enter the cavity, while the intracellular gate is closed. Following ATP hydrolysis, the NBD dimer dissociates, and the intracellular gate opens and releases the substrate into the cell.

In addition to the uptake of cobalamin, M. tuberculosis also scavenges iron from the host using siderophores called mycobactin and carboxymycobactin. The heterodimeric $\mathrm{ABC}$ transporter IrtA — IrtB (IrtAB) was previously shown to import iron-loaded carboxymycobactin. A distinct feature of IrtAB is the presence of a siderophore-interaction domain (SID) fused to the amino terminus of IrtA, which has been implicated to have a role in the release of iron inside the bacterial cell. In the second paper, Seeger and colleagues determined the IrtAB structure of Mycobacterium thermoresistibile. They showed that the transporter assumes an inward-facing conformation. They also identified a partially collapsed inward-facing substrate-binding cavity, which may represent the low-affinity state after substrate release. They went on to show that purified IrtAB reconstituted in liposomes mediates siderophore uptake, and the SID is capable of reducing the siderophore-bound iron. Finally, the authors showed that Irt $\mathrm{AB}$ preferentially imports mycobactin over carboxymycobactin, and they speculate that there may be an additional import system for the latter. On the basis of the findings, the model suggests that IrtAB imports iron-bound mycobactin across the inner membrane, where it is reduced by the SID and released inside the cell.

In sum, both studies provide insights into the substrate import mechanisms of unusual ABC exporters and the possibility to pursue those as drug targets to develop novel antibiotics.

Andrea Du Toit

ORIGINAL ARTICLES Rempel, S. et al.

A mycobacterial $A B C$ transporter mediates the uptake of hydrophilic compounds. Nature https:// doi.org/10.1038/s41586-020-2072-8 (2020)| Arnold, F. M., Weber, M. S. \& Gonda, I. et al. The ABC exporter IrtAB imports and reduces mycobacterial siderophores. Nature https://doi.org/10.1038/ s41586-020-2136-9 (2020) 